Fernando C. Barros 1 Cesar G. Victora 1 Elaine Tomasi 1 Bernardo Horta 1 Ana Maria Menezes 1 Juraci A. Cesar 1 Ricardo Halpern 1 Maria Teresa Olinto 1 Cora Luiza Post ${ }^{2}$ Juvenal S. D. Costa 1 Flávio S. Menezes 1 Maria del Mar Garcia 3 J. Patrick Vaughan 4

\section{Saúde materno-infantil em Pelotas, Rio Grande do Sul, Brasil: principais conclusões da comparação dos estudos das coortes de 1982 e 1993}

\author{
Maternal and child health in Pelotas, \\ Rio Grande do Sul, Brazil: \\ principal conclusions from the 1982 \\ and 1993 cohort studies
}

1 Departamento de Medicina Social, Faculdade de Medicina, Universidade Federal de Pelotas. Caixa Postal 464, Pelotas, RS, 96001-970, Brasil.

2 Faculdade de Nutrição, Universidade Federal de Pelotas. Caixa Postal 464, Pelotas, RS 96001-970, Brasil.

3 Escuela Andaluza de Salud Publica, Campus Universitario de Cartuja. Ap. Correos 2070, 18080 Granada, España.

${ }^{4}$ Department of Public Health and Policy, London School of Tropical Medicine and Hygiene. Keppe Street, London WCIE7HT, U. K.

\begin{abstract}
This paper summarizes the main findings of the Pelotas birth cohort studies of 1982 and 1993. There was a reduction in the number of births from 6,011 in 1982 to 5,304 in 1993, which was not evenly distributed, as there were around 1,000 fewer births in the poorest groups and 300 more in the high-income strata. Nutritional status of the mothers also varied in the decade, with an increase of $3.5 \mathrm{~cm}$ in mean height and $3.9 \mathrm{~kg}$ in mean weight at the beginning of pregnancy. Despite such improvements, the proportion of low birthweight increased from 9.0\% in 1982 to $9.8 \%$ in 1993. Preterm births and intrauterine growth retardation also increased. There was a reduction in perinatal mortality from 32.2/1.000 births in 1982 to 22.1/1.000 births in 1993. Nutritional status at 12 months of age varied according to the indicator: there was a slight increase in low height for age in 1993, whereas a reduction was observed in the prevalence of low weight for age and weight for height. The infant mortality rate dropped from 36.4/1.000 live births in 1982 to 21.1/1.000 in 1993.

Key words Epidemiology; Infant Mortality; Perinatal Mortality; Breastfeeding; Nutrition
\end{abstract}

Resumo Este artigo resume os principais achados dos estudos das coortes materno-infantis de Pelotas em 1982 e 1993. Houve uma redução no número de nascimentos, de 6.011 em 1982 para 5.304 em 1993, fato não distribuído de forma eqüitativa entre os diferentes grupos de renda familiar, ocorrendo entre as mulheres de baixa renda cerca de 1.000 nascimentos a menos do que em 1982. O grupo com renda mais elevada contribuiu com um aumento de cerca de 300 nascimentos. A situação nutricional das mães apresentou variações na década, com um aumento médio de 3,5 cm em estatura e 3,9 kg no peso no início da gestação. Apesar destas melhoras, a proporção de recém-nascidos de baixo peso aumentou para 9,8\% em 1993 (9,0\%, 1982). Observou-se um aumento na incidência de nascimentos pré-termo e de retardo de crescimento intra-uterino assim como uma redução nos coeficientes de mortalidade perinatal-32,2/1.000 em 1982 e 22,1/1.000 em 1993. A situação nutricional aos 12 meses de idade apresentou comportamentos distintos, com um discreto aumento do déficit de comprimento/idade em 1993, e uma redução de quase 50\% nos déficits de peso/idade e peso/comprimento. O coeficiente de mortalidade infantil decresceu de 36,4 /1.000 nascidos vivos em 1982 para 21,1/.000 em 1993.

Palavras-chave Epidemiologia; Mortalidade Infantil; Mortalidade Perinatal; Amamentação; Nutrição 


\section{Introdução}

O objetivo do artigo final desta série é refletir sobre os principais resultados da comparação dos estudos de 1982 e 1993, levantar algumas indagações com respeito a achados cujos significados ainda não estão totalmente apreendidos, e apontar algumas recomendações e novas linhas de investigação. Devido à amplitude das mudanças que ocorreram no cenário político, econômico e social, durante o período coberto pela investigação, tentativas de explicar o significado das mudanças encontradas nos indicadores de saúde, considerando somente as informações coletadas nas próprias pesquisas, podem ser difíceis. Não obstante, pensamos que estas reflexões são válidas, principalmente pela escassez de estudos deste tipo na literatura.

Os estudos das coortes de nascimento de Pelotas em 1982 e 1993 mostraram, em primeiro lugar, que é factível realizar, no Brasil, investigações epidemiológicas com este delineamento e dimensão. Nos dois anos estudados, todos os nascimentos hospitalares ocorridos em uma cidade de porte médio foram investigados e os resultados destes estudos mostraram ser extremamente úteis, pois revelaram informações que não estavam disponíveis e que são fundamentais para o entendimento do processo saúde-doença e para a priorização de ações preventivas de saúde.

O estudo da coorte de 1982 produziu, além de numerosas publicações na literatura internacional, um livro em português e espanhol A Epidemiologia da Desigualdade -, que enfeixou os principais resultados do estudo, redigidos de forma acessível para profissionais de diferentes níveis. Do ponto de vista institucional, houve também ganhos de grande importância, pois a pesquisa aglutinou um grupo de pesquisadores no Departamento de Medicina Social da Universidade Federal de Pelotas, que formaram o núcleo de um Centro de Pesquisas Epidemiológicas, o que, posteriormente, ensejou a criação de um Mestrado em Epidemiologia.

A seguir serão feitos comentários sobre os achados principais dos artigos que formaram esta série.

\section{Crescimento populacional e número de nascimentos}

A comparação de coortes de recém-nascidos de Pelotas em 1982 e 1993 evidenciou uma redução no número de nascimentos, de $6.011 \mathrm{em}$ 1982 para 5.304 em 1993. Por outro lado, dados dos Censos revelam que a população urbana da cidade de Pelotas, que era de 209.074 em 1980, aumentou para 265.193 em 1991. Para a população feminina na faixa etária de 15 a 44 anos, este aumento foi de 53.943 em 1982 para 66.493 em 1993. Houve uma queda importante na taxa de fertilidade (nascidos vivos pela população feminina em idade fértil) - de 109,6 em 1980 para 78,9 em 1991.

Estas reduções importantes de fertilidade, que também têm sido documentadas em outras regiões do País (Patarra, 1995), parecem ser devidas a uma maior utilização de métodos contraceptivos, incluindo formas definitivas, como a laqueadura tubária. De fato, em um inquérito de base populacional realizado na cidade em 1992, encontrou-se que $18,4 \%$ das mulheres com idade entre 20 e 49 anos haviam sido esterilizadas (Costa, 1993). Por outro lado, não é possível descartar que parte deste decréscimo de nascimentos possa ter ocorrido por um maior número de abortamentos, uma vez que esta informação é de difícil comprovação, tendo em vista seu caráter ilegal.

\section{Situação sócio-econômica das famílias}

Quando se comparam as características das mulheres que tiveram filhos em 1982 com aquelas de 1993, as diferenças são muito marcadas. A redução de 707 nascimentos em 1993 não foi distribuída de forma eqüitativa entre os diferentes grupos de renda familiar. De fato, entre as mulheres de baixa renda (menos de três salários mínimos mensais de renda familiar) ocorreram cerca de 1.000 nascimentos a menos do que em 1982, tendo o grupo com renda mais elevada contribuído com um aumento de cerca de 300 nascimentos. Portanto, estas informações levam a supor que a utilização de métodos contraceptivos, definitivos ou não (e/ou abortamentos), durante a década, foi maior entre as famílias de menor renda familiar. O real significado destas diferenças, entretanto, não pode ser inferido de forma definitiva, tendo em vista que a década estudada caracterizou-se por uma intensa inflação, e o valor do salário mínimo pode ter variado de forma significativa.

Outro indicador utilizado para comparar a situação sócio-econômica das duas coortes foi a distribuição por classe social, muito embora esta informação, para a coorte de 1982, tenha sido coletada somente em 1986. De qualquer forma, este indicador apresenta-se, como já era esperado, mais robusto, ou seja, as modificações ocorridas durante a década são bem 
mais discretas e caracterizam-se por um aumento da pequena burguesia tradicional, enquanto nota-se um pequeno esvaziamento do proletariado típico, da burguesia e da nova pequena burguesia.

Estes resultados são interessantes e sugerem que, entre as famílias que tiveram filhos nestes anos, houve um aumento daquelas que tiveram que procurar novas profissões, não-assalariadas, para garantir a sua subsistência.

\section{Características biológicas maternas}

Quanto às características biológicas das mães de 1982 e de 1993, as diferenças são marcantes e apontam para uma seleção, em 1993, de mães em melhor situação nutricional. Assim, enquanto em 1982 a estatura média das mulheres era $156,4 \mathrm{~cm}$, e $11 \%$ das mães tinham menos de $150 \mathrm{~cm}$, em 1993 a estatura média aumentou $3,5 \mathrm{~cm}$, passando para $159,9 \mathrm{~cm}$. Neste ano, somente 4,6\% das mães tinham menos de 150 $\mathrm{cm}$. Da mesma forma, o peso médio materno no início da gravidez foi substancialmente maior em 1993 - 62,1 kg, quando comparado com aquele de $1982-58,2 \mathrm{~kg}$. O ganho de peso durante a gravidez foi discretamente maior em 1982 (11,8 kg) do que em $1993(11,6 \mathrm{~kg})$, mas esta diferença não alcançou níveis de significação estatística $(\mathrm{p}=0,07)$.

A marcada melhora da situação nutricional das mães de 1993 parece ser um reflexo de sua melhor inserção social, pois não ocorreram diferenças significativas na idade ou paridade destas mães que pudessem sugerir outras razões. Da mesma forma, o tempo transcorrido entre os estudos e a magnitude da diferença em estatura não permite supor que se trata somente de um fenômeno de crescimento secular. É também importante notar que, em todos os grupos de renda familiar, assim como em todos as classes sociais, houve melhoras significativas em peso e estatura.

Apesar das melhores condições nutricionais das mulheres que tiveram filhos em 1993, a avaliação de risco obstétrico, obtida a partir de um conjunto de variáveis utilizadas nos estudos perinatais ingleses e adaptadas para as condições brasileiras, não mostrou diferenças significativas. A proporção de mulheres consideradas como de alto risco obstétrico foi de $12,4 \%$ em 1982 e 13,6\%\% em 1993. Este critério valoriza, de forma especial, o passado reprodutivo das mães, razão pela qual as diferenças nutricionais das duas coortes não foram capazes de mostrar uma redução no risco.

\section{Assistência à saúde: pré-natal, parto e cobertura vacinal infantil}

Durante a década ocorreram muitas alterações no sistema de prestação de serviços de saúde, já descritas nesta publicação. Fundamentalmente, a rede de prestação de atenção pré-natal foi bastante ampliada, tendo sido assumida pelo sistema de atenção primária. Quanto ao atendimento ao parto, ampliaram-se os serviços de residência médica, o que garantiu maior presença do médico na sala de parto, e foram implantadas unidades de atendimento intensivo ao recém-nascido, que não existiam em 1982.

A cobertura pré-natal foi melhor em 1993, com uma média de atendimentos por mulher de 7,6, em relação a 6,6 em 1982. A proporção de mulheres que receberam menos de cinco consultas pré-natais foi de $23,4 \%$ em 1982, tendo esta cifra caído para 17,8\% em 1993. Portanto, apesar dos progressos obtidos na década, cabe ressaltar que, neste centro urbano, que possui uma rede bastante ampla de serviços de saúde, quase 1/5 das mulheres grávidas ainda não fazem o número mínimo de consultas prénatais preconizadas pela Organização Mundial de Saúde.

Quanto à assistência ao parto, 61\% dos nascimentos ocorridos em 1982 foram atendidos por médico, sendo que as mulheres de mais alto risco tiveram menor assistência médica (57\%) do que aquelas de baixo risco (70\%). Em 1993, a atenção médica foi ampliada, com $88,3 \%$ das mulheres atendidas por médico durante o parto. No entanto, o fenômeno dos cuidados inversos permaneceu ativo, pois a assistência médica ao parto continuou sendo maior entre mulheres de baixo risco (92\%) do que entre aquelas de risco elevado (87\%), ao contrário do que seria indicado. Parece, portanto, que a ampliação dos serviços de atenção ao parto, proporcionada em grande parte pelas residências médicas, aumentou o atendimento médico, mas permaneceu não levando em consideração critérios de risco ou necessidade.

No que se refere às operações cesarianas, por outro lado, a prevalência aumentou de 27,7\% em 1982 para 30,5\% em 1993. Este incremento foi notado em todos os grupos sócioeconômicos, particularmente na população de alta renda, onde estes partos cirúrgicos elevaram-se de 46,7\% em 1982 para 55,4\% em 1993.

Do ponto de vista de imunizações infantis, o aumento das coberturas das vacinas Sabin e tríplice confirmam a efetividade das repetidas campanhas nacionais que têm sido realizadas. 


\section{Baixo peso ao nascer e nascimentos pré-termo}

Talvez o achado mais intrigante deste estudo tenha sido o aumento da proporção de crianças de baixo peso ao nascer, especialmente as nascidas pré-termo, tendo em vista a melhor situação sócio-econômica e nutricional das mães, comentadas anteriormente. A proporção de recém-nascidos de baixo peso (menos de $2.500 \mathrm{~g}$ ), que era de 9,0\% em 1982, aumentou para 9,8\% em 1993. Esta diferença não é estatisticamente significativa $(\mathrm{p}=0,2)$, porém, quando se ajustam, através da utilização de regressão logística, os efeitos da nutrição materna (peso no início da gravidez e altura) e situação sócio-econômica (renda familiar e educação materna), o risco de nascimento com baixo peso torna-se 33\% maior em 1993 ( $\mathrm{p}<0,01)$.

$\mathrm{O}$ aumento na incidência de nascimentos pré-termo, de 5,6\% em 1982 para 7,5\% em 1993, assim como o aumento na ocorrência de retardo de crescimento intra-uterino $(15,0 \%$ em 1982 e $17,5 \%$ em 1993) foram significativos e necessitam ser entendidos, para que ações preventivas possam ser tomadas. O aumento no baixo em peso ao nascer, em 1993, foi decorrente tanto da maior proporção de nascimentos de crianças pré-termo com peso adequado para a idade gestacional, como de crianças nascidas a termo, com retardo de crescimento intra-uterino. Possíveis causas para o aumento de pré-termos poderiam ser interrupções das gestações antes de seu término normal, o que poderia ocorrer por ações médicas, como cesarianas ou induções de parto, ou por infecções maternas que desencadeassem o trabalho de parto pré-termo. As análises até agora realizadas não implicam cesarianas ou induções de parto como responsáveis por este aumento. Por outro lado, a ocorrência de infecções maternas, como as do trato urinário, que foram muito comuns em 1993, não foi investigada em 1982, o que impede qualquer tipo de comparação. Quanto ao retardo de crescimento intrauterino em crianças nascidas a termo, as causas para o aumento em 1993 são ainda mais difíceis de serem entendidas, considerando a melhor situação nutricional das mães neste ano.

\section{Mortalidade perinatal}

Apesar do aumento do baixo peso ao nascer já relatado, houve uma redução bastante importante nos coeficientes de mortalidade perinatal (CMP), tanto no componente fetal como no neonatal precoce. Enquanto em 1982 ocorre- ram 194 óbitos perinatais, com um CMP de 32,2/1.000 nascimentos, em 1993 houve 117 mortes neste período, caindo o CMP para $22,1 / 1.000$ nascimentos. A marcada redução da mortalidade fetal poderia ser explicada por melhores condições de saúde materna e melhor atendimento pré-natal. Quanto aos melhores índices de mortalidade neonatal precoce, podem ter colaborado para tal a ampliação da atenção médica e a criação de unidades de tratamento intensivo de recém-nascidos, que eram inexistentes em 1982. Como já era esperado, tanto em 1982 como em 1993, a mortalidade de crianças pré-termo foi cerca de 2,5 vezes maior do que entre crianças com retardo de crescimento intra-uterino.

Um achado que deve ser esclarecido é por que a mortalidade na primeira semana de vida entre crianças de baixo peso pertencentes a famílias de baixa renda é mais de três vezes superior à das crianças pertencentes a famílias mais afluentes. Assumindo que as unidades de tratamento intensivo neonatal tratam de forma equânime crianças de diferentes classes sociais, a razão mais provável é que as patologias apresentadas pelas crianças de famílias pobres sejam mais severas.

Finalmente, cabe salientar a enorme queda no sub-registro de óbitos perinatais ocorrida entre 1982 e 1993. Este é um exemplo de que a primeira forma para resolução de um problema é a constatação de sua existência, pois o progresso obtido foi fruto da discussão de suas causas, após o estudo de 1982, com as autoridades de saúde do Município.

\section{Amamentação}

Percebe-se que houve um aumento na proporção de crianças sendo amamentadas ao seio nos primeiros meses de vida em 1993, quando se compara com as cifras de 1982. Com um mês de idade, por exemplo, a proporção de crianças recebendo leite materno predominante aumentou de $65 \%$ em 1982 para $72 \%$ em 1993. Esta melhora nos padrões de amamentação é mais notável aos três meses de idade, quando de cerca de $1 / 3$ de crianças amamentadas em 1982, a proporção aumentou para 53\% em 1993. As diferenças nos padrões de amamentação se desvanecem aos seis meses, fase em que os padrões são idênticos na década.

É importante que sejam identificadas quais as razões que levaram a uma melhoria nos níveis de amamentação entre crianças menores de seis meses, idade em que o leite materno é mais importante, para que mais ênfase ainda 
seja dada a estas medidas. É muito possível que as campanhas de promoção do aleitamento que ocorreram durante a década, assim como a delimitação da propaganda de leites em pó, tenham colaborado para mudar a conduta das mães com relação a esta prática. Será também importante avaliar como tem sido a evolução da amamentação em outros locais do País.

\section{Situação nutricional}

A evolução da situação nutricional aos 12 meses de idade, durante a década, apresentou comportamentos distintos, conforme o indicador antropométrico utilizado. Assim, houve um discreto aumento do déficit de comprimento/idade, que evoluiu de 5,3\% em 1982 para $6,1 \%$ em 1993. Por outro lado, houve uma importante redução de quase 50\% na prevalência de déficits de peso/idade (5,4\% em 1982 e $3,8 \%$ em 1993) e peso/comprimento.

Estas variações de situação nutricional na década devem ser analisadas à luz das modificações ocorridas com o peso ao nascer e a idade gestacional, tendo em vista que o crescimento de recuperação ("catch-up growth") é bastante diverso entre crianças nascidas prétermo e aquelas com retardo de crescimento intra-uterino. Devem-se considerar também as variações que aconteceram nos padrões de amamentação e nas condições ambientais, que podem ter afetado a morbidade infantil, especialmente a ocorrência da diarréia. A diminuição observada nas hospitalizações por esta doença permite supor que houve uma redução, pelos menos nos casos severos, que pode ter influenciado o padrão de crescimento infantil.

A diferente evolução nos indicadores nutricionais também pode reforçar a idéia de que os determinantes do ganho de peso e do crescimento linear não sejam exatamente os mesmos.

\section{Hospitalizações}

O estudo de hospitalizações reveste-se de alguma complexidade, pois seus índices revelam não somente a proporção de pacientes com morbidade severa, que necessitam ser tratados em hospital, mas também a disponibilidade destes serviços e o acesso aos mesmos pelos usuários.

No ano de 1982, 19,6\% das crianças foram hospitalizadas pelo menos uma vez durante o primeiro ano de vida. Esta cifra foi bastante semelhante - 18,1\% - em 1993. Chama a atenção que, nos dois anos estudados, as crianças de sexo masculino foram hospitalizadas com maior freqüência do que as de sexo feminino, fato que tem sido descrito também em outras populações. Na Espanha, por exemplo, a proporção de hospitalizações entre crianças abaixo de quatro anos de idade é quatro vezes mais elevada em meninos (EASP, 1994).

No que se refere à evolução das hospitalizações em Pelotas durante a década, é também interessante notar que houve uma discreta redução nas proporções de crianças hospitalizadas em famílias de baixa renda, enquanto que, entre aquelas de renda mais elevada, as hospitalizações foram um pouco mais freqüentes em 1993. Quanto às hospitalizações conforme o peso ao nascer, manteve-se, como já era previsto, uma forte relação inversa entre este indicador e as hospitalizações. No grupo de crianças com peso abaixo de $2.000 \mathrm{~g}$ ao nascer, houve um acentuado aumento das hospitalizações em 1993, o que provavelmente se deve a um artefato de diferentes critérios utilizados para definir uma hospitalização infantil.

\section{Mortalidade infantil}

Ocorreram progressos significativos para a mortalidade infantil, assim como para a mortalidade perinatal. Entre os recém-nascidos de 1982 ocorreram 215 óbitos infantis, enquanto que para os de 1993 este número foi de 111 . Uma redução de 104 mortes de crianças deve ser comemorado como um progresso importante. O coeficiente de mortalidade infantil, que em 1982 era de 36,4 / 1.000 nascidos vivos, foi reduzido para 21,1/1.000, um decréscimo de $42 \%$. É também alentador observar que, na camada mais carente da população, o coeficiente de mortalidade infantil caiu de 80/1.000 em 1982 para 33/1.000 em 1993.

Por outro lado, é importante ressaltar que este progresso observado nos coeficientes não reduziu as diferenças observadas entre os grupos sociais. Assim, embora a mortalidade tenha sido reduzida em todos os grupos em 1993, crianças de famílias de baixa renda (igual ou inferior a um salário mínimo mensal) apresentaram coeficientes de mortalidade seis vezes mais elevados do que aquelas oriundas de famílias com renda superior a 10 salários mínimos mensais. É interessante salientar que reduções importantes dos coeficientes de mortalidade infantil nas últimas décadas têm sido documentadas em outras regiões do País (Simões \& Monteiro, 1995; Monteiro \& Nazário, 1995), e que a análise das reduções conforme a 
causa do óbito e o estrato social são fundamentais para o entendimento do fenômeno.

Vale a pena ressaltar que a redução de óbitos pós-neonatais foi ainda superior (57\%) à dos óbitos neonatais (29\%), o que parece indicar que nesta área urbana do Sul do País, em meio a uma crise econômica que perpassou a década, foi possível diminuir de maneira significativa as mortes infantis de causas evitáveis, através de imunizações e melhoria de problemas ambientais, como condições de moradia, suprimento de água e saneamento. De fato, ao observar-se a redução de óbitos infantis conforme a causa, nota-se que os decréscimos mais importantes ocorreram justamente no grupo das infecções.

\section{Considerações finais}

É certo que, em todos os tópicos abordados nos diferentes artigos deste suplemento, novas análises se fazem necessárias para o melhor entendimento das alterações ocorridas nos indicadores de saúde na década estudada. De forma geral, houve ganhos de saúde maternoinfantil no período estudado, com acentuadas quedas nos coeficientes de mortalidade. Esta série demonstra a importância de estudos de base populacional para adicionar informações que permitam comparar a evolução dos indicadores de saúde e as razões para as diferenças encontradas.

\section{Referências}

COSTA, J. S. D., 1993. Utilização de Serviços de Saúde Ambulatoriais em Pelotas. Dissertação de Mestrado. Pelotas: Faculdade de Medicina, Universidade Federal de Pelotas.

EASP (Escuela Andaluza de Salud Publica), 1994. Evaluación del Programa de Salud Materno Infantil de Andalucia. Granada: EASP.

MONTEIRO, C. A. \& NAZÁRIO, C. L., 1995. Declínio da mortalidade infantil e eqüidade social; o caso da cidade de São Paulo entre 1973 e 1993. In: Velhos e Novos Males da Saúde no Brasil. A Evolução do País e de Suas Doenças. (C. A. Monteiro, org.), pp. 173-185, São Paulo: Hucitec/NUPENS/Universidade de São Paulo.

PATARRA, N. L., 1995. Mudanças na dinâmica demográfica. In: Velhos e Novos Males da Saúde no Brasil. A Evolução do País e de Suas Doenças. (C. A. Monteiro, org.), pp. 61-78, São Paulo: Hucitec/ NUPENS/Universidade de São Paulo.

SIMÕES, C. C. S. \& MONTEIRO, C. A., 1995: Tendência secular e diferenças regionais da mortalidade infantil no Brasil. In: Velhos e Novos Males da Saúde no Brasil. A Evolução do País e de Suas Doenças. (C. A. Monteiro, org.), pp. 153-156, São Paulo: Hucitec/NUPENS/Universidade de São Paulo. 